and in every case $I$ found that intemperance, and sleeping in ill-ventilated rooms, had produced a loss of appetite and consequent debility. During this debility they inhaled the poisonous gas, and its effects upon them was rapidly fatal.

"One boatman in particular, a very fine man, stated that he had been drinking for'two or three days, that he had not had any appetite, and that all he had tasted for some hours before being attacked was a few ripe cherries! On examination of this man after death, the cherry pulp was found in his stomach as fresh and undeconposed as if it had been but just swallowed. It was thought by several that the cherries produced the disease, but I do not entertain any such opinion; on the contrary, I may here venture to offer an opinion as to the cherry pulp being found undecomposed in the stomach after it had been so long swallowed. I imagine that the disease had already paralyzed the powers of digestion, consequently the pulp of the cherries remained, as it were, in a non-vital sac, and atmospheric air being excluded, fermentation was also prevented. At that time there was a great outcry against vegetables; but $I$ do not believe that vegetables render any one more liable to the disease (where:vegetables do not generally disagree, when taken with a proper quantity of animal food.

"During the autumn of 1832, a very active and zealous physician fell a sacrifice to the disease in my neighbourhoed: In his case, the liability to the disease was brought on by great bodily fatigue, during his attendance upon cholera patients; and by the anxiety attendant upon his profession; he lost his appetite, and was incapable of taking sufficient nourishment to afford him vital energy adequate to resist the disease, which proved fatal in a few hours. It is necessary to avoid all debilitating causes, whether it be too much drink or too much solid food, for gluttony will produce debility as well as drunkenness. It would be well to advise the public thus: 'Let your meals be plain, wholesome, nourishing, and taken at regular periods; avoid long fasting, and all food likely to disagree with you; avoid as much as possible residing in damp situations, and in the neighbourhood of foul drains; observe cleanliness in your person and in your dwelling; use warm clothing, and keep the functions of the body in action by a due amount of exercise; and if after all these precautions you should be attacked, let me warn you against tampering with yourself by taking quack medicines, (for I have proved that there is no specific for cholera, but send for your qualified medical attendant without loss of time, as every moment is precious.'

"A word now with-my medical brethren as to the treatment of cholera. The first object to be attained is to enable the blood to recover its lost oxygen. How is this to be done I. have found calomel and tartar emetic to have both succeeded in restoring the patient in the following cases:-

"A widow, ared forty-eight, living in a damp situation in a crowded street in the bottom of a valley; was in the collapsed stage when seen. Treabment: The room was cleared of everything but the bed, the window was opened, and ventilation established. A hot nitro-hydrochloric acid bath was procured, in which the patient was placed for half an hour; when taken out, she was well rubbed with cloths and flannel. A pill, containing two grains of calomel, was given every five or ten minutes, and a tablespoonful of the following mixture every half hour or hour:-Sesquicarbonate of soda, a drachm; carbonate of potass, two drachms; water, eight ounces; sedative solution of opimm, a drachm; hydrocyanic acid, (Scheele?) twenty minims. Mix. 240 grains of calomel were given in about thirty-two hours, (a great part of which I myself administered, and two bottles of the mixture; and at the end of that period warmth was restored to the body, the cramps had ceased, and bile was observed in the last de jection. From that period the patient rapidly recovered. The rationale of this treatment I conceive to be, the supply of oxygen from the decomposition of the calomel by the potass which, being passed directly to the liver, entered quickly into the circulation; the skin also received a great stimulus from the nitro-hydrochloric acid baths (three of which were used) and the friction.

"Another casa of cholera in the collapsed stage was that of a boy, fourteen years old. In this case tartar-emetic was used, with nitro-hydrochloric acid baths and friction, and certainly the recovery was far more rapid. Three grains of tartar-emetic were dissolved in four ounces of water, and an ounce was given every three hours. After the third dose, vomiting was established, and bile was mixed with the ejected fluid. From that time the boy rapidly recovered, and he sat up the next day. The operation of the tartar-emetic I conceive to be principally upon the lungs and skin. If we observe the breathing of cholera patients, they appear as if they had no power fully to inflate the lungs; and it appears to me pro. bable that the sjoasm of the body cxtends to the air-tubes, closing them up, and preventing the atmospheric air from penetrating to the air-ceils; consequently, no oxygen is obtained, by breathing, until the spasm of the bronchial tubes is removed. The tartar-emetic effects this removal quickly, and by aiding the atmospheric air to penetrate to the aircells, thereby ensures a supply of oxygen to the stagnant, or nearly stagnant blood. This desired end might perhaps be effected by inhaling oxygen gas with atmospheric air, or by the nitrous oxide gas.

"I have selected these two cases merely to point out the difference of treatment, each being successful; but certainly from the rapid manner in which tartar-emetic effected its purpose, I should feel inclined to place greater confidence in it than in any other medicinal agent. We must not, however, neglect any adjunctive measures, such as the nitrohydrochloric acid baths, friction, ventilation, \&c., any or all of which might be deemed necessary to suit an individual case, according to the judgment of the medical attendant."

A correspondent has addressed to us some remarks on the treatment of cholera, suggesting the use of the following remedies-viz., (in his own words, "Internally: carbonated fluids, or solutions of the most volatile compounds of carbon, or even pure carbon, with aromatics. - Externally: warm baths, impregnated freely with sulphuretum potasso c. sulphure; and frequent odoration, or smelling of the carburet of sulphur, or, perhaps, oleum cassire; and the carburet of sulphur, in some form of liniment, (not oily,) may be well rubbed into the course of the spine, with copious diluents and external warmth.

"The grounds for the above deductions (he adds) are too extensive to be here submitted, or at present. The plan being derived from a survey of natural laws; it is recommended for trial."

On the Famine Fever, as it occurred at Croydon, Surrey, during. July, August, and September, 1847.

By George Botromlex, Esq., M.R.C.S.E., Croydon, SURGEON TO CROYDON UNION.

A considerable immigration of Irish to the neighbourhood of Croydon always occurs during the summer months, occasioned by the harvest, and the hop-gathering in the adjacent county. of Kent; and about the middle of June, 1847, it took place to a much greater extent than usual. Nearly all the-Irish patients who applied for parochial medical relief at that and subsequent periods had evidently suffered from the privations of famine in the winter and spring of 1847, and had been compelled to make great physical exertions to obtain the means of accomplishing their journey to this country. The result was, an influx of fever cases to the Infirmary during: two of the summer months, which appeared to be greatest at the beginning of August; and in all, 100 patients were treated in the Infirmary for this fever, of whom three died, and seventy-nine received out-door medical relief, amongst whom one death occurred.

"The patient, on presenting himself, nsually complained of great prostration of strength, pains in all the bones, constant thirst, no disposition to eat, and usually pain in the head. His appearance was haggard; countenance very anxious; eyes sumken; general condition emaciated; the pulse small and compressible, varied from 90 to 120 ; tongue covered with a clear white coat, and exhibited nervous tremor. The patient had always experienced rigors at the commencement. of the attack; in some cases, constipated bowels, but fre quently diarrhoea. The history of the case elicited the facts, of the patient having slept abroad for some previous nights under a hedge or in a ditch, and of his having walked some miles during the same or previous days. On his appearance at the Infirmary, therefor it might be calculated that for six or eight days he had struggled with a condition of great depression of the vital powers. The symptoms already. enumerated would continue with little alteration for a week, during which time the skin was pungently hot and dry, and the urine scanty and high coloured. After the primæ viæ had been acted upon by laxatives, mild mercurials and diam phoretics were administered, usually in the following form :Chloride of mercury, two grains; powdered opium, half a grain; potassio-tartrate of antimony, one-sixth of a grain. Mix for a pill. One pill might be given for four nights consecutively, or perhaps one night and morning, as the case demanded. In others, the mercury with chalk, combined with the compound ipecacuanha powder, seconded the alte 
rative and sedative effects which were required. Saline draughts were given frequently, free sponging of the body was used with much advantage, and toast and barley-water were supplied liberally for drink. Diet to be low. The most usual complication was a cerebral affection. Cold applications were constantly renewed to the head, which was often shaved. A blister to the nape of the neck was extremely efficacious, if the case became urgent. At the end of the first week after admission, a kind of crisis appeared. After several hours' profuse perspiration, the patient's symptoms decidedly improved; the tongue began to clean, the pains in the limbs to abate, and all that was complained of was extreme weakness. More substantial diet seemed to be indicated, and in another day or two the patient would earnestly request it. If the symptoms continued to improve under more nourishing regimen, as in the majority of instances was the case, for a few days all promised exceedingly well; but almost invariably, by the end of another week, a sudden change would take place, the tongue become as coated as ever, and all the original symptoms would present themselves, and apparently in as much force as at first. The recurrence to low diet of course immediately followed: the relapse was treated as the primary attack, though usually it proved less obstinate, in point of duration. The recovery would then take place very slowly. The longest remaining sequence was want of muscular power, and most patients, though looking quite well, found, on their dismission from the Infirmary, a much greater prostration of the vital powers than either they or any casual observer would have suspected. None, I should think, were able to engage in agricultural labour during the season. At the end of the first week reforred to above, just as the system was throwing off the first onset of the febrile attack, when the arterial action was diminishing, no local congestions presenting, and the digestive functions were seemingly in more healthy condition, the crisis would frequently result in a different course. After two or three days of apparent quiescence, which might be presumed, on the whole, to be something like general improvenent, a yellowish line would appear down the centre of the tongue; dryness and a slightly cracked appearance followed; the pulse became weaker, and gradually sank to 60 or 70 ; the countenance wore a more anxious expression; and there was disinclination to food. On the administration of port wine or brandy, as the state of the case might seem to indicate, these symptoms would perhaps gradually subside, and the recovery might take place withont the relapse, as in the foregoing instances; but in a few cases, complete typhus ensued, with the usual symptoms of great nervous depression. These were constantly treated with concentrated nourishment and stimulants, and when the power of swallowing was in aberance, strong beef-tea injections supported Nature until her powers rallied. Ammonia and quinine, with small opiates, were administered according to circumstances; and with close watching and constant attention, the third week would nearly bring the patient through the severity of the attack, which then left him in an exceedingly weak condition. Here especially-although the rule applies generally to every case-the greatest care was necessary in directing the advance towards convalescence. That it should be sure, it was absolutely necessary to be also slow.

In reviewing the general character of the fever in question, it is worthy of remark, that the complications were those of the head and the mucous lining of the intestinal canal. To three patients (out of 179) jaundice occurred, terminating fatally in one case. In one or two instances only was there any tendency to chest affection, as a consequence of the fever. From these details, it will be apparent that the form of fever described approaches nearly to that known as the "simple continued," the "Synochus of Callen." But a strong tendency invariably existed towards a termination in one of a low nervous type or typhoid. Keeping this in view, the indications were plain-viz, to be as sparing of antiphlogistic treatment as the case would allow, discarding the use of the lancet altogether. Even in the administration of mercury, antimony, and purgative medicines, great care was necessary, not to be too liberal in the use of depressants, and to commence the exhibition of nutritious diet, however small the quantity, as soon as the stomach was enabled to perform its functions. The first tendency to exhaustion was promptly met by stimulants cautiously and gradually afforded, according to the urgency of the symptoms, with strict attention to the cerebral affection, as evidenced by a comatose condition of the patient, insensibility of the pupil, subsultus tendinum, \&c., which were combated by means of cold applications and counter-irritation. With unremitting attention to ventilation and cleanliness I believe little or no danger of infection existed, and the patients were distributed indiscriminately among those suffering from other diseases. Had separate wards been devoted to the fever patients alone, I have no doubt the results would have been far less favourable; under the plan adopted the other patients have not suffered. Two out of six nurses who were engaged in constant attendance were attacked, and exhibited the type of disease described, in rather a severe form, but this is by no means surprising if we consider the very arduous character of their engagements at this period. I acted on the principle of providing full and stimulating diet for them, and I recommended to the visiting clergymen the only precaution which I and those who assisted me adopted -namely, the taking a glass of wine and a biscuit before entering the wards. Not the slightest fever symptoms were manifested in those brought into contact with the sick.

"I have no hesitation in attributing so small a mortality4 in 179 -chiefly to the unlimited supply of stimulus and concentrated nutriment which $I$ had it in my power to afford, and to the constant exertions and watchful attention with which the much-respected matron of the Infirmary and our experienced nurses seconded the directions of the medical at tendants."

\section{Description of an Improved Tourniquet.}

By H. V. MLALAN, M.D.

Dr. Malan urges his claim as the inventor of an improved tourniquet, now in use in Great Britain and on the Contivent, and he subjoins a description of the instrument, as given in his "Dissertation for Obtaining the Diplomas of Doctor of Medicine, Surgery, and Midwifery, in the University of Tubingen, in 1839." This tourniquet, he states, was then publicly examined and tried before competent judges, and a description of it was soon afterwards published in a medical journal, under the sanction of the rector of Tubingen University.

Dr. Malan states-" During my subsequent travels I found it lectured upon in Paris, Lyons, and Edinburgh, by various professors of surgery. My friend the late Mr. Liston was in the habit of using it.

"Finding it, therefore, known and valued in the profession, I feel anxious not to allow it to remain unconnected with my name; and the more so, as it is frequently mentioned in medical publications and journals, without the acknowledgments ordinarily due to the inventor."

A technical description of "Malan's tourniquet" accompanies Dr. Malan's communication; but it would be unsuitable in thi - place, and without many attendant engravings. The chief advantages of the instrument are stated to be these:-

"As the key can be adapted to either end of the instrument, it can be placed on either side of the limb, below or above, as required. The instrument, as a single glance will prove, is simple and strong; it can be put on, fixed, and tightened, with littie trouble or effort on the part of the operator, and, owing to its two double screws, with much power and rapidity; the compression is especially made on the bloodvessel, by means of the pad, and its very construction makes it lie low and close to the limb, so that not only it cannot be upset by the sudden collapse of the muscles after the amputation, but can be used in any position or applied to any part of the body, and is not in the way of any garment slipped over it; it is compact, small, and safe. As the key may be remored when the instrument is once fixed, no patient can, in any case, free himself from its grasp. Such, $I$ believe, is the general opinion, since it has been in use, now upwards of eight years.

Upper Seymour-street West, Portman-square."

\section{3ncbieins.}

Medicine an Art, and its Truths to be Attained. By Thoras Shaprer, M.D., \&c. London: Churchill. 1848. Pamphlet. pp. 31 .

THIS is an Address read recently at the opening meeting of the Library of the Exeter Dispensary, and of the Devon and Exeter Pathological Society. The writer, using Dr. Whewell's definition, places medicine among the arts -and indeed it will be admitted by all that practical medicine is an art, however, we believe we may, in another point of view, deem medicine a science. The greater part of this address is taken up by a consideration of the fallacies committed 